\title{
Evidencia sobre la movilidad económica intergeneracional en Nicaragua
}

\author{
Francisco Mendoza Tijerino* y Carlos Narváez Silva
}

\section{Recibido: agosto de 2013 / Aprobado: octubre de 2013}

En base a las Encuestas de Medición del Nivel de Vida 2005 y 2009 se estimó la elasticidad económica intergeneracional para el caso de Nicaragua, medida que permite apreciar el grado de movilidad económica entre generaciones. Se identificó un gran contraste entre los niveles de ingresos generados por los más ricos y los más pobres, obteniéndose un coeficiente de Gini de 0.489 , superior al coeficiente del ingreso estimado por el Instituto Nacional de Información de Desarrollo debido, en parte, a la forma de construcción de la variable ingreso. La desigualdad persistente a través de una generación se analiza bajo el enfoque de divergencia en las capacidades y libertades de los individuos, permitiendo así relacionarla directamente con el grado de desarrollo económico. Se utilizó el método de variable instrumental y datos de corte transversal agrupados en el tiempo para estimar el valor de la elasticidad intergeneracional en Nicaragua, que se encuentra entre 0.25 y 0.33 , indicando que la desigualdad en la distribución de ingresos perdura con el paso de una generación.

Palabras clave: movilidad económica intergeneracional / pobreza / desigualdad / desarrollo

\section{Introducción}

Al analizar las características de la pobreza en Nicaragua es posible evidenciar un escenario en donde los pobres se encuentran destinados a seguir siendo pobres y engendrar su condición a lo largo del tiempo. Esta particularidad de persistencia en el nivel de desarrollo de una generación dista de ser una característica única de la economía nicaragüense y es distinguida por Sachs (2012) cuando indica: "Muy

* Departamento de Investigación del Instituto de Investigación y Desarrollo NITLAPÁN-UCA. Rotonda Rubén Dario 150 mts. al oeste, Managua, Nicaragua. Correo electrónico: franciscomt91@hotmail.com

** Ingeniero y economista. Consultor independiente. Correo electrónico: carlnarsil@yahoo.com 
a menudo, niños que nacen en condiciones de pobreza terminan siendo adultos pobres".

Al hacer una visión retrospectiva de la desigualdad en la distribución de los ingresos en Nicaragua conforme a las Encuestas de Medición del Nivel de Vida (EMNV) publicadas por el Instituto Nacional de Información de Desarrollo (INIDE), se presentan medidas de desigualdad persistentes con el paso de los años: se pasa de un coeficiente de Gini de 0.55 en 2001, a uno de 0.46 en 2009. Si se obvian los cambios metodológicos que presentan las EMNV en este intervalo de tiempo, la discrepancia en ingresos que se exhibe en la economía nacional aún es alta, revelando la existencia de una brecha muy marcada entre ricos y pobres.

Es evidente que la disparidad en la distribución de los ingresos es una problemática aguda, ya que implica, bajo el enfoque de Sen (1999), una disparidad en las 'capacidades' y 'libertades' de los individuos. Sin embargo, el gran contraste es solamente preámbulo para un análisis más agudo a nivel intergeneracional: la mejora o deterioro en el nivel de vida que tienen los individuos en relación al nivel de vida que exhibían sus padres una generación atrás y su grado de transmisión o persistencia entre generaciones.

Bajo la perspectiva de Sen (1999) el análisis intergeneracional se vuelve atractivo, ya que se buscaría realizar un estudio relativo sobre la transmisión de las diferencias en las capacidades existentes de los agentes económicos en relación a una generación atrás (o varias generaciones si la información existente lo permite).

El presente artículo plantea el inicio del análisis de la movilidad intergeneracional en la economía nicaragüense utilizando la información de las encuestas de hogares, las preguntas que se buscan responder mediante la estimación de la elasticidad intergeneracional son:

- ¿ $\quad$ ¿Un hijo que nace en condiciones de pobreza se encuentra destinado a seguir siendo pobre en su edad adulta, o existe la posibilidad de lograr "escalar" en el escalafón de ingresos?

- ¿Hasta qué punto el ingreso de un individuo en su edad adulta depende de lo que el padre lograba generar una generación atrás?

Tomando en cuenta la correlación positiva existente entre mejoras en los niveles de ingresos, e indicadores como salud, educación y alimentación, además de las mayores capacidades de capitalizar oportunidades en los rangos más altos de ingresos, se busca encontrar qué persistencia existe entre la calidad de vida de un padre con la de un hijo en Nicaragua mediante la estimación de la elasticidad económica intergeneracional, medida que captura el estado de movilidad económica entre generaciones.

\section{Revisión de literatura}

La literatura concerniente al tema de la movilidad económica intergeneracional (MEI) se encuentra en crecimiento. Nace fundamentalmente por la preocupación ante la desigualdad en la distribución de los ingresos en Estados Unidos, las 
economías europeas y las latinoamericanas, y cómo esta desigualdad ha persistido en el tiempo. Es necesario conocer qué aproximación se ha utilizado para obtener medidas fiables de movilidad intergeneracional y qué se entiende sobre la MEI analizando los estudios existentes.

Mazumder (2012) establece que la medida que se ha utilizado para medir la MEI, la elasticidad intergeneracional, es una mesura con un "vistazo hacia atrás", en donde la elasticidad del ingreso del hijo con respecto al del padre describe el grado de movilidad que tienen los hijos para subir o bajar en la escalera social en relación a su progenitor.

El autor sostiene que una menor elasticidad intergeneracional del ingreso sugiere menor persistencia de desigualdad en la distribución de la renta y mayor movilidad, mientras que una mayor elasticidad intergeneracional del ingreso es asociada con una menor movilidad intergeneracional. En este plano, se indica que la elasticidad para Estados Unidos se establece entre 0.5 y 0.6, lo que significa alta persistencia y poca movilidad.

Mazumder (2012) resalta la importancia que los padres han dado a la inversión en educación, siendo la creación de capital humano uno de los puntos clave detrás de la movilidad económica intergeneracional, aunque cabe resaltar que no es el único.

La relevancia de la estimación de la elasticidad intergeneracional se basa en los resultados obtenidos por el autor en su análisis. La movilidad económica intergeneracional se ha mantenido prácticamente constante desde 1990 en Estados Unidos, lo que es indicador de pocas mejoras en las condiciones de vida entre las generaciones analizadas, dado que persiste la desigualdad en la distribución de ingresos.

La persistencia en el valor de la elasticidad intergeneracional del ingreso es indicador de bajo aprovechamiento ( $\mathrm{y} / \mathrm{o}$ acceso) de oportunidades. Esto se debe a que las condiciones económicas presentadas por los padres no son las mismas presentadas por los hijos y, sin embargo, persiste la desigualdad en los ingresos de la siguiente generación. El autor indica que la elasticidad intergeneracional provee una imagen aproximada de las oportunidades existentes en una sociedad. Ampliando este razonamiento, la elasticidad intergeneracional tiene el significado de un correcto y oportuno aprovechamiento de tales oportunidades.

Si se analiza más de cerca, la MEI hace referencia a movilidad en ingresos provenientes de actividades productivas en una economía de mercado. El uso del ingreso, independientemente del padre y del hijo, ha sido ampliamente discutido en la literatura del desarrollo económico como uno de los aspectos necesarios (no suficiente) para el desarrollo económico. Es clara la relación directamente proporcional entre en los ingresos y el acceso a educación, sanidad y alimentación (Debraj, 1998), por lo que su uso se vuelve favorable para examinar las mejoras en el desarrollo ${ }^{1}$ que puede tener, en promedio, un hijo con respecto a su padre en la economía.

1 Vistas bajo la perspectiva de Sen (1999), estas mejoras en el desarrollo implican una mayor capacidad de acceso a servicios básicos, mayor capacidad de ejercer libertad y mayor capacidad de decisión en relación a una generación anterior. Es decir, las mejoras en las 'capacidades' y 'libertades'. 
DeParle (2012) analiza lo referido a las oportunidades. El autor revela que una de las razones por las cuales existe un bajo y persistente grado de movilidad en Estados Unidos es porque los pobres al nacer pobres tienen desventajas en comparación a las clases medias y/o altas. Se trata de desventajas en acceso a educación y a inversión en capital humano: prácticamente una nueva forma que expresa una trampa de pobreza.

Bajo la misma perspectiva, Corak (2006) reafirma que la variable ingreso se encuentra altamente correlacionada con el nivel de educación, y que éste último es fundamental en el tema de la movilidad económica intergeneracional. Se parte de que los padres establecen sus decisiones de incidencia en el bienestar de los hijos en función del ingreso de largo plazo que se tiene (o ingreso permanente), y no necesariamente en el ingreso esporádico. Esta incidencia en el bienestar de los hijos puede tomar la forma de inversión en educación y en la generación de condiciones necesarias para potenciar el capital humano del hijo.

Corak (2006) indica que, si bien es cierto que la elasticidad de movilidad es una medida apropiada que captura el grado de oportunidad que tienen los hijos con respecto a sus padres, este indicador no es el principal lineamiento para el planteamiento de acciones de políticas de desarrollo económico debido a que al utilizar la elasticidad de movilidad no queda claro cuál debería de ser la meta de las políticas a implementar. Así mismo, el autor realiza un recorrido de las aproximaciones que otros han realizado a la temática de la MEI. Establece un contraste entre la aproximación teórica realizada por Solon (2004) y la de Mayer y Lopoo (2004) indicando que para el primero los mayores retornos a la educación tienden a mantener estancada - e incluso a disminuir - la movilidad económica entre las generaciones.

Esto puede entenderse tomando en cuenta que los retornos a la educación, se asume, son determinados en una economía de mercado: similar retorno a la educación tendrá un rico que un pobre, por lo que las oportunidades derivadas de la inversión en capital humano que tiene el rico sobre el pobre se podrían mantener estables, generando así una persistencia en la desigualdad.

El contraste se realiza analizando el razonamiento de Mayer y Lopoo (2004), el cual sostiene que las diferencias en los retornos a la educación no cambian la igualdad en las oportunidades per se, sino que alteran los costos económicos y sociales de oportunidades discrepantes. Esto implica que puede existir una mayor capitalización de los retornos a la educación por parte de los individuos que tienen mayor estatus social ${ }^{2}$.

Finalmente, Núñez y Miranda (2007) utilizan la técnica de variables instrumentales bi-muestrales propuesta por Arellano y Meghir (1992) y Angrist y Krueger (1992) como solución a la problemática de no poseer información de los ingresos de los padres e hijos en pares. La estimación resulta en una elasticidad intergeneracional que se encuentra en el intervalo de 0.52 y 0.67 para Chile, evidenciándose persistencia intergeneracional.

\footnotetext{
2 Personas con mayores niveles de ingresos se encontrarian, bajo esta perspectiva, con mayores probabilidades de aprovechar y capitalizar los retornos a la educación en comparación al aprovechamiento de los individuos con menores ingresos.
} 


\section{Materiales y método}

Para la estimación econométrica y el análisis de las variables estrechamente relacionadas con la movilidad económica intergeneracional se utilizaron las EMNV 2005 y 2009 elaboradas por el INIDE. Se realizaron estimaciones utilizando la técnica de Mínimos Cuadrados Ordinarios, Mínimos Cuadrados en dos Etapas, y Estimación a través de Variable Instrumental, complementadas con el método de combinación de corte transversal (Pooling data).

La ventaja del uso de las EMNV 2005 y 2009 radica en que es una encuesta en la que se reportan los ingresos de los hogares y los individuos derivados de actividades productivas, regalos/remesas, rentas y demás fuentes de ingreso, a pesar de no ser una encuesta dirigida especialmente a la captura de dicha variables. Sin embargo, a pesar de la ventaja de haberse reportado el ingreso de los hogares y/o individuos en la encuesta a utilizar, estas variables no son aptas para su uso en estimaciones econométricas pues presentan diferencias sustanciales en sus frecuencias, que deben ser tomadas en cuenta previo al análisis.

El INIDE (2011a) establece y calcula su ingreso a partir de dos grandes fuentes:

i. Ingreso proveniente de actividades de trabajo

ii. Ingreso proveniente de actividades diferentes al trabajo

En la presente estimación se hace uso del ingreso derivado de fuentes de trabajo, dejando por un lado los regalos y fuentes que no se derivan necesariamente de actividades productivas. Por lo tanto, la variable ingreso construida y usada en las estimaciones econométricas del artículo incluye para ambas encuestas (2005 y 2009): salario neto de su trabajo primario, decimo tercer mes e ingresos por trabajos por cuenta propia, empleadores y/o miembros de cooperativas. Esta construcción del agregado ingreso busca obtener remuneraciones que sean lo más estables posible debido a que para la estimación de la elasticidad intergeneracional lo ideal es la obtención de los salarios de largo plazo de los padres e hijos, y la información disponible no logra capturar a cabalidad dicha condición.

\section{Modelo teórico}

Para la estimación de la elasticidad económica intergeneracional se usó el modelo propuesto por Solon (2004). Se partió de una modificación hecha por el autor al modelo teórico planteado por Becker y Tomes (1979).

Los supuestos básicos del modelo se basan en que el hogar i tiene un padre de la generación $\mathrm{t}-1$ con un hijo del periodo $t$, en donde el padre divide su ingreso después de impuestos entre su propio consumo () e inversión en el capital humano del hijo y donde no existe herencia de activos hacia los hijos, cumpliendo la restricción presupuestaria:

$$
(1-\tau) \mathrm{y}_{\mathrm{i}_{-1}}=\mathrm{C}_{\mathrm{t}_{-1}}+\mathrm{I}_{\mathrm{i}_{\mathrm{t}_{-1}}}
$$

El capital humano del hijo queda representado por:

$$
\mathrm{h}=\theta\left(\mathrm{I}_{\mathrm{i}_{-1}}+\mathrm{G}_{\mathrm{t}_{-1}}\right)+\mathrm{e}_{\mathrm{t}}
$$


Donde representa la inversión pública en el hijo y la dotación de capital humano del hijo.

Es de esperarse que la dotación del hijo se encuentre correlacionada con la dotación del padre, por lo que se presenta la relación:

$$
\mathrm{e}_{\mathrm{t}}=\delta+\lambda \mathrm{e}_{\mathrm{t}-1}+\mathrm{v}
$$

Finalmente, el ingreso del hijo es determinado por una función log-lin:

$$
\log y_{t}=\mu+\mathrm{ph}_{\mathrm{t}}
$$

Dadas estas relaciones, se asume que el hogar, al decidir orientar su ingreso al consumo o a la inversión en el hijo, maximiza una función de utilidad CobbDouglas

$$
\mathrm{U}=(1-\alpha) \log \mathrm{C}_{\mathrm{t}_{-1}}+\alpha \log \mathrm{y}_{\mathrm{t}}
$$

Suponiendo que se cumplen las condiciones presentadas anteriormente, al encontrar la función objetivo en términos de la variable y obteniendo la demanda óptima de ésta, el modelo teórico indica que los padres se encuentran más inclinados a invertir en el capital humano de los hijos mientras mayor sea el ingreso.

Haciendo uso de algebra se puede llegar a la ecuación que indica que la movilidad intergeneracional, al igual que el ingreso del padre, puede verse influenciada por la inversión pública en capital humano del hijo:

$$
\log y_{t} \cong \mu+\theta p \log \left[\frac{\alpha \theta p(1-\tau)}{1-\alpha(1-\theta p)}\right]+\theta p \log y_{t_{-1}}+\theta p\left[\frac{G_{t_{-1}}}{(1-\tau) y_{t_{-1}}}\right]+p e_{t}
$$

Lo que finalmente se deriva en la ecuación a estimar:

$$
\log y_{\mathrm{t}} \cong \mu *+[(1-\gamma) \theta \mathrm{p}] \log \mathrm{y}_{\mathrm{t}_{-1}}+\mathrm{pe}_{\mathrm{t}}
$$

En donde:

$$
\mu *=\mu+\varrho \theta p \log \left\{\frac{\alpha \theta p(1-\tau)}{[1-\alpha(1-\theta p)]}\right\}
$$

Y $[1-\gamma) \theta p]$ viene dado por la caracterización de la inversión pública que presenta la relación:

$$
\left[\frac{G_{t_{-1}}}{(1-\tau) y_{t_{-1}}}\right] \cong \varrho-\gamma \log y_{t_{-1}}
$$

\section{Nicaragua: Movilidad Económica Intergeneracional}

Desde 1993 se realiza periódicamente en Nicaragua la EMNV, una encuesta que permite estimar índices de desigualdad para la distribución de los ingresos y el consumo, como por ejemplo el conocido coeficiente de Gini ${ }^{3}$ que captura qué tan disímil es la distribución de los mismos.

\footnotetext{
3 Se puede hacer uso, igualmente, de indices como Mehran, Kakwani y Piesch, fácilmente calculables en softwares estadísticos además de estadígrafos como varianza y desviación estándar, los cuales capturan la dispersión en una distribución.
} 
Al revisar los coeficientes de Gini oficiales para Nicaragua, desde inicios de la década del 2000 los niveles de desigualdad en el país han sido persistentes, pasando de un coeficiente de Gini de 0.55 en 2001 a uno de 0.46 en 2009. Por consiguiente, se sabe que existe una gran disparidad entre los ingresos generados por el quintil más alto y el quintil más pobre, manteniéndose esta brecha por casi una década.

Sin embargo, las diferencias metodológicas de las encuestas de hogares dificultan capturar la capacidad de los individuos de subir o bajar en el escalafón social debido a que las observaciones en las encuestas han cambiado, lo que conlleva a no conocer el grado de 'movilidad' que pueden tener los agentes económicos para pasar desde una condición de pobreza a una condición de mayor bienestar.

En este sentido, el análisis de la movilidad económica intergeneracional es necesario porque permite examinar, primero, si la desigualdad en la distribución de los ingresos que predomina en la economía nicaragüense responde a factores circunstanciales o si es que depende de causas más de fondo como los escenarios iniciales a los que los hijos desde el nacimiento se enfrentan (pobreza familiar, trabajos poco remunerados de los padres, área de residencia rural, etc.) y que vienen a determinar un estancamiento en el nivel de desarrollo de su generación. Segundo, permite examinar el grado de 'oportunidades' que impera en la economía.

\subsection{Desigualdad en distribución de los ingresos en Nicaragua}

Analizar la desigualdad en la distribución de los ingresos implica analizar una desigualdad de resultados. La gran disparidad en la distribución del ingreso es resultado, en gran parte, de contrastes en las oportunidades a las cuales tienen acceso los agentes económicos. Precisamente aquí se evidencia la estrecha relación entre la desigualdad de resultados y la movilidad económica intergeneracional, dado que esta última permite conocer el grado de oportunidades que surgen en la economía de mercado y a las cuales se enfrentan los agentes económicos en sus relaciones de intercambio. Estimaciones oficiales y propias de coeficientes de desigualdad en Nicaragua expresan contrastes preocupantes que han mantenido el espacio que separa a los ricos de los pobres. Pero no se conoce aún el grado de movilidad que existe año tras año.

La desigualdad en la distribución de los ingresos significa una disparidad en la 'capacidad' de acceder a recursos, el hecho de que un segmento de la población posea mayor capacidad de acceder a recursos debido a su mayor nivel de ingreso muestra que la economía de mercado que se ha moldeado en el país no distribuye equitativamente su renta.

Consecuentemente, se parte de la percepción de que un crecimiento económico que reparta sus beneficios equitativamente entre toda la población es positivo (Debraj, 1998) aunque aún no exista consenso entre la posición de perfilar la repartición equitativa de la riqueza entre la población como una condición suficiente para garantizar crecimiento económico.

Los diferenciales en la capacidad de acceder a recursos determinan de manera fundamental el logro de las libertades de agencia y libertades de bienestar en el sentido de Sen (Bastiaensen et al., 2011). Básicamente la libertad de bienestar implica el 'poder elegir' de cara a un objetivo y la libertad de agencia implica el 
'poder cambiar' los parámetros que rigen la elección, lo que involucra el poder para generar el cambio de los parámetros de cara a los objetivos planteados.

Contar con niveles de desigualdad en ingresos (no necesariamente en riqueza), ante la ausencia de acceso a educación, alimentación y demás servicios básicos, limita el poder de cambio que puede tener un agente económico durante su desarrollo, evitando así un pleno alcance de bienestar y mejoramiento en el nivel de vida.

Tomando como referencia la EMNV 2009 se estima que el coeficiente de Gini para el ingreso anual neto más decimo tercer mes de los individuos que laboran en su trabajo primario es de 0.489 , casi tres puntos superior al coeficiente reportado por el INIDE para el ingreso oficial en el año 2009. Cabe resaltar que el INIDE incluye en su estimación el trabajo secundario y otras fuentes de ingreso, como el ingreso en especie.

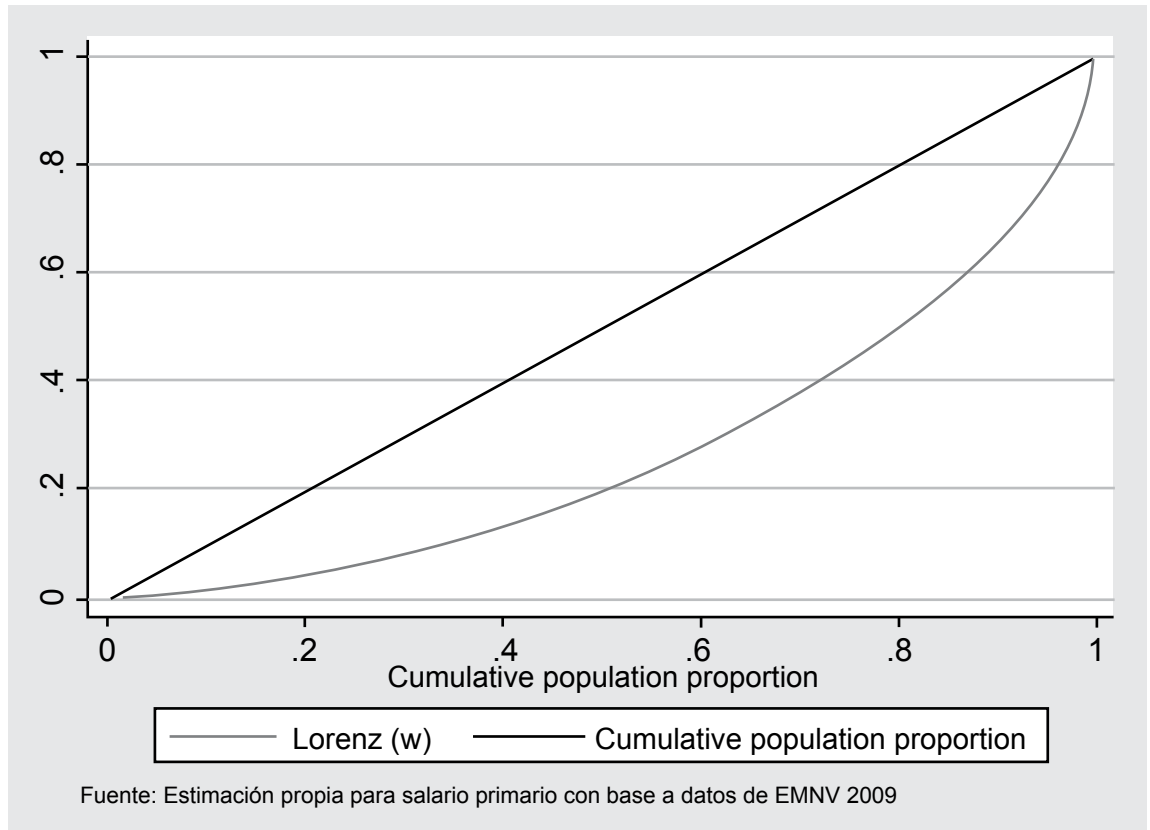

Ilustración 1. Estimación de Curva de Lorenz para el trabajo primario según la EMNV 2009

En la Ilustración 1 se puede apreciar la Curva de Lorenz para los ingresos anuales (w) de los individuos que laboran en su trabajo primario a nivel de economía. ${ }^{4}$ La gráfica indica que no se presenta igualdad en la distribución de ingresos dado que ambas curvas no coinciden en los 45 grados.

Por el contrario, existe una marcada disparidad en la distribución de los ingresos y, analizando ambos ejes, ordenadas y abscisas, es posible ver a primera vista que el 10\% más rico (en términos de capacidad de generación de ingresos estimados y no de riqueza acumulada) de la población captura aproximadamente el $30 \%$ de la 
renta estimada para el trabajo primario. Sumado a esto se puede ver que el 5\% más rico posee un ingreso al menos 79 veces superior al ingreso de las personas que se encuentran en el percentil más pobre de la distribución de los ingresos.

Cuadro 1. Estimación de percentiles: ingreso anual para trabajadores con trabajo primario

\begin{tabular}{|l|l|l|}
\hline Percentiles & Ingreso $(\mathrm{C} \$)$ & Número de veces el percentil más pobre \\
\hline $1 \%$ & 1,400 & 1 \\
\hline $5 \%$ & 4,000 & 2.85 \\
\hline $10 \%$ & 7,200 & 5.14 \\
\hline $25 \%$ & 15,600 & 11.14 \\
\hline $50 \%$ & 28,800 & 20.57 \\
\hline $75 \%$ & 48,000 & 34.28 \\
\hline $90 \%$ & 77,356 & 55.25 \\
\hline $95 \%$ & 111,184 & 79.41 \\
\hline $99 \%$ & 264,000 & 188.57 \\
\hline
\end{tabular}

Estimación de los autores en base a la EMNV 2009.

En promedio, un trabajador con un trabajo primario ganaba alrededor de 40,918 córdobas al año, pero la utilización del promedio de los ingresos puede llevar a una visión errónea ya que este estadígrafo se ve alterado por los valores extremos de una distribución no normal. Hacer uso de la mediana es más conveniente pues captura el ingreso de la mitad de la distribución, permitiendo obtener una visión más aproximada de la clase media en el país. En 2009 la mediana del ingreso anual era de 28,800 córdobas, un valor muy inferior al promedio presentado inicialmente.

Tales diferencias en la distribución del ingreso son difíciles de explicar. Una idea generalmente utilizada y aceptada es que la desigualdad es el resultado de las fuerzas del mercado (Stiglitz, 2012). La teoría económica dominante establece que en condiciones competitivas el pago al factor productivo, es decir el salario, será igual a su contribución o, como lo establece la teoría microeconómica, su productividad marginal.

Utilizando estimaciones basadas en la EMNV 2009 se presenta la Ilustración 2 , donde se puede ver que, tomando como razonamiento que los salarios se pagan en base a la productividad marginal del factor trabajo, es difícil comprobar una relación clara. Al utilizar como variable los años de educación y asumiendo que conforme se tengan más años de educación un trabajador será más productivo, se puede apreciar que no existe una marcada relación positiva en Nicaragua. El coeficiente de correlación entre los años de educación y los ingresos del trabajo primario es muy bajo, $33.75 \%$, lo que significa poca asociación entre las variables en cuestión. 


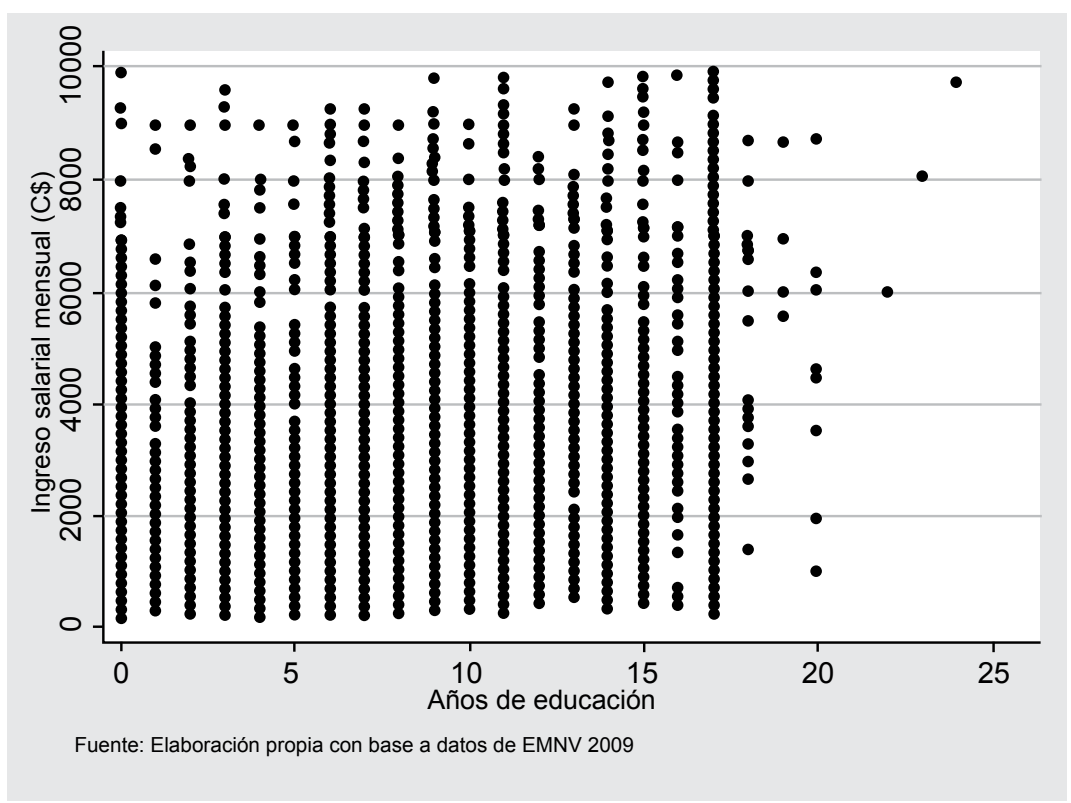

Ilustración 2. Relación entre ingresos del trabajo primario y años de educación para ingresos mensuales de entre 200C\$ y 10,000 C\$

Dicho esto surge un llamado a la precaución, ya que la desigualdad en la distribución de los ingresos no permite tener una visión completa de la severidad de sus consecuencias en el grado de desarrollo en una economía. Se recalca que es una medida de resultado, desigualdad que se da al final de un proceso pero que en ausencia de movilidad económica puede generar estancamiento en el nivel de progreso de los hogares.

Es el análisis intergeneracional el que permite obtener una buena medida del grado de oportunidades que se han generado en la economía: analizando el ingreso del padre en relación al ingreso del hijo, y constatando la existencia (o no existencia) de persistencia en los ingresos, se puede obtener una aproximación al grado de movilidad económica a la que se enfrenta una generación.

\subsection{Movilidad intergeneracional}

La desigualdad económica y el análisis intergeneracional, además de ser temas de interés por sí mismos y de estar relacionados estrechamente entre sí, conllevan a juicios que sobrepasan el campo de estudio de la economía. Debraj (1998) lo indica cuando establece que la riqueza de los padres y su 'acceso a los recursos' pueden hacer que dos niños no comiencen en igualdad de condiciones.

\footnotetext{
5 Paes de Barro, Ferreira, Molina y Saavedra (2008) relacionan las "oportunidades" con el acceso a servicios básicos como educación de calidad, salud, nutrición, seguridad y vivienda, y al grado de desigualdad en el acceso por parte de diferentes grupos.
} 
Que exista alta movilidad intergeneracional (elasticidad intergeneracional baja) puede atribuirse a la existencia de igualdad de oportunidades en el acceso a recursos ${ }^{5}$, o visto desde otra perspectiva, a que la capacidad de generación de ingreso (que en última instancia viene a determinar la salida de la pobreza) ${ }^{6}$ no dependa de una condición inicial exógena o del contexto en el que se encuentra inmersa una persona, sino de su capacidad y empoderamiento en sus actividades económicas, y de la decisiones y esfuerzo de cada individuo.

El análisis de la movilidad económica intergeneracional radica en estudiar qué tanto influye $\mathrm{n}$ las condiciones iniciales en el desempeño económico (fundamentalmente medido por el ingreso) de la generación posterior. La literatura referente al tema muestra que la elasticidad económica intergeneracional, es decir, la medida del grado de movilidad entre generaciones existente en una economía, describe cómo los ingresos relativos cambian a través de una generación, y por lo tanto, permite obtener una noción del grado de oportunidad que existe en la economía.

Se supone que la existencia de movilidad implica que si dos padres distintos en una generación atrás poseen niveles de ingresos distintos, uno muy debajo y otro muy arriba del promedio de ingresos, el ingreso de los hijos en la siguiente generación no va a verse influenciado por este contexto en el que nacieron y se desenvolvieron, permitiendo así que quien nació en condiciones de pobreza pueda lograr obtener mayores ingresos y tenga la posibilidad de mejorar su calidad de vida. En este sentido, la capacidad de obtener mayores ingresos es independiente de los ingresos del padre (situación inicial), y depende del aprovechamiento de las oportunidades de acceso a servicios básicos como salud, educación de calidad, nutrición, etc.

Lo ideal sería tener economías en donde las diferencias en los ingresos de las generaciones no se transmitan en el tiempo a las generaciones siguientes. La persistencia en la desigualdad en la distribución de los ingresos a través de generaciones puede vincularse también a que el tipo de empleo que se genera en la economía no ha cambiado mucho; trabajadores pobres, usualmente del sector agropecuario en Nicaragua (generador de alrededor del $31 \%$ del empleo), con bajo o nulo nivel de escolaridad suelen incorporar a las generaciones venideras a las mismas tareas de baja productividad en ausencia de oportunidades de acceso a escuelas o educación desde pre-escolar.

Dicho esto surge la pregunta: ¿Es deseable tener una alta movilidad intergeneracional? La respuesta es que depende del tipo de economía, y depende de si se analizan países ricos o pobres, ya que la movilidad económica intergeneracional indica la capacidad de subir o bajar en el escalafón de ingresos generación tras generación. Para países en vías de desarrollo, como Nicaragua, en donde la pobreza general azota al $42.5 \%$ de la población según información oficial (INIDE, 2011b) con una muestra mayoritariamente urbana y con desigualdad persistente, sí se debería desear una mayor movilidad. Esto implicaría que una persona nacida pobre tendría la oportunidad de no serlo en su adultez. 


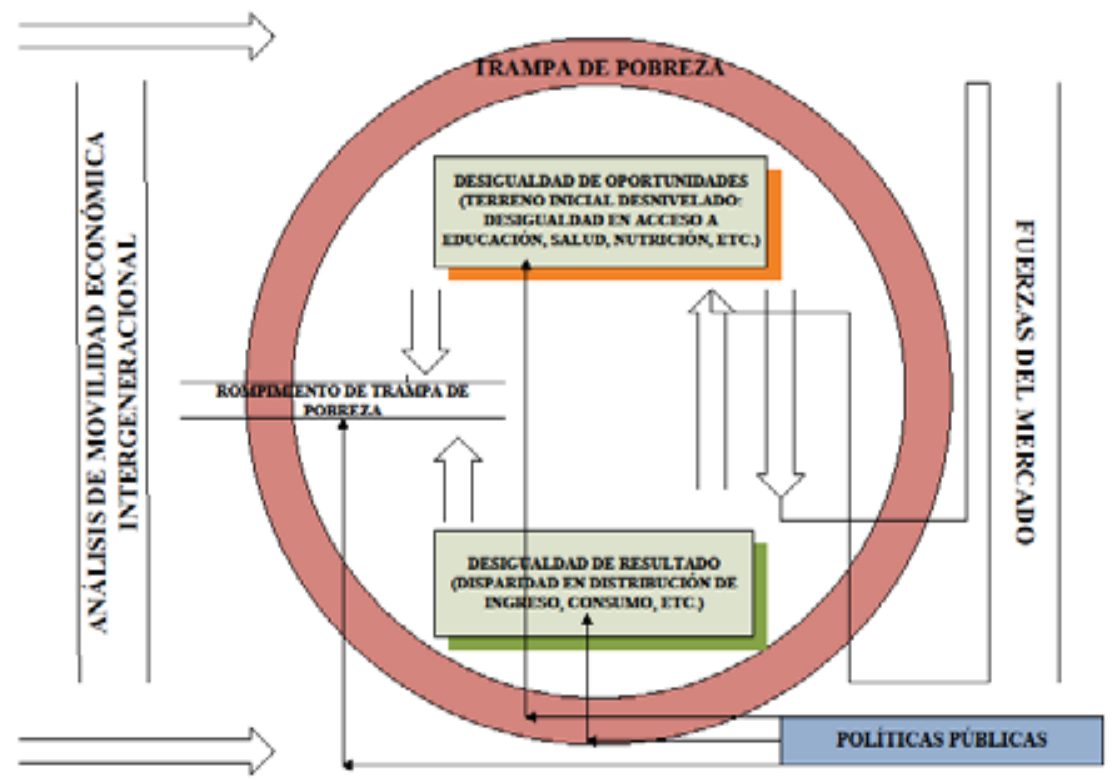

Ilustración 3. Marco de análisis de la movilidad económica intergeneracional Fuente: Elaboración propia

Finalmente, se puede ver el marco de análisis planteado para el mejor entendimiento de la movilidad económica intergeneracional, que puede servir para abordar el tema desde un punto de vista analítico. Claramente las fuerzas del mercado no garantizan la salida de una trampa de pobreza, condición en donde la movilidad económica intergeneracional se retroalimenta a través de la persistencia de la desigualdad en la distribución de ingresos. Las políticas públicas vienen a nivelar el terreno a través de equidad en las oportunidades, las cuales rompen el círculo de la pobreza. De la misma manera, el abordaje de ambos tipos de desigualdades, de resultado y de oportunidades, debe ser de manera sistemática y coherente para lograr el planteamiento de políticas efectivas y de manera eficiente.

\subsection{Estimación econométrica}

La construcción teórica de la temática en cuestión indica que el padre de la generación $t-1$ se enfrenta a su restricción presupuestaria y busca maximizar su bienestar derivado de consumo corriente e inversión en capital humano del hijo, lo que lleva a la siguiente función a estimar econométricamente:

$$
\log y_{t}=\mu *+\delta \log y_{t-1}+e_{t}
$$

La estimación de la elasticidad intergeneracional para los hijos en relación al padre toma en cuenta el ingreso derivado de actividades productivas, esto es: salario neto anual y decimo treceavo mes de los trabajadores de sexo masculino que laboran como empleados/obreros, jornaleros/peones, empleadores, trabajadores por cuenta propia y miembros de cooperativas y que tienen un trabajo en el que han laborado 
más de cinco meses. Se toma el salario del jefe del hogar (independientemente de que sea padre o madre) como variable independiente asumiendo que es éste quien realiza las decisiones de compra o inversión en la familia.

Se analiza la movilidad intergeneracional únicamente para los hijos de sexo masculino para evitar sesgar las estimaciones econométricas que puedan derivarse de la característica discriminatoria del mercado de trabajo nicaragüense - estimaciones propias sugieren que las mujeres perciben ingresos sustancialmente inferiores a los ingresos de los hombres, incluso en condiciones socioeconómicas similares.

Por otro lado, se obvian los trabajos esporádicos, definiéndolos como trabajos en los que se ha laborado por menos de seis meses. Se espera que a mayor tiempo trabajando en una actividad económica (o trabajo específico) existan mayores probabilidades de mantenerse trabajando en ésta.

Así mismo, para las estimaciones econométricas se hace uso de los hijos del hogar que tengan más de 17 años de edad (18 años o más) bajo el supuesto de que a esta edad la independencia (de decidir en qué sector, cuánto tiempo trabajar, etc.) de los hijos es superior a la independencia que se tiene a la edad oficial de entrada al mercado laboral, que es de 14 años.

Para la estimación econométrica se toman en cuenta los siguientes puntos:

1) Solon (1992) indica que el uso de un solo año para la estimación de la MEI (como es el caso del uso de la EMNV 2009) puede tener efectos de subestimación de la transmisión intergeneracional debido a la presencia de componentes momentáneos en el ingreso. Lo ideal sería obtener los ingresos de largo plazo de los hijos y los padres, y hasta que el proceso de movilidad haya terminado. No obstante, la información disponible obliga a restringir la variable ingreso al salario primario reportado para personas que han trabajado por más de cinco meses en sus labores reportadas.

2) $\mathrm{Al}$ realizar la estimación con las restricciones tomadas en cuenta resultan pocas observaciones en relación a las observaciones que originalmente generaban ingresos (se debe depurar para los hijos que efectivamente tengan padres que generen ingresos y que trabajen, que sean hombres y que tengan una edad mínima definida).

3) En la encuesta utilizada sólo se toman en cuenta los hijos que viven en el hogar al momento de reportarse los datos por parte de las familias, por lo que puede existir un sesgo ya que el hijo o padre pueden verse incentivados a no buscar un trabajo más remunerativo porque el otro ya posee uno. En este sentido, lo deseable sería ver a los hijos que son también jefes de hogar o que conforman otra familia en otro lugar, sin embargo esta información no es posible de obtener mediante las encuestas de hogares. A esta debilidad se le llamará en el resto del documento como 'debilidad de ubicación'.

4) Las estimaciones de elasticidad intergeneracional presentadas muestran sesgo derivado de autocorrelación y la variable dependiente incluida como regresora y rezagada un periodo.

La estimación se realiza utilizando como variable dependiente e independiente los valores predichos de una ecuación de Mincer (Heckman, Lochner \& Todd, 2005) con el fin de resolver el problema derivado de la 'debilidad de ubicación'. La lógica 
detrás de esta estrategia consiste en obtener una variable que haga referencia a los ingresos salariales (más decimotercer mes) que no dependan del hecho de habitar en el mismo hogar. Esto significa obtener una medida de salario que depende de variables exógenas, planteándose de ésta manera, un modelo de determinantes del salario.

La teoría econométrica (Wooldridge, 1999) indica que al tener estos problemas de endogeneidad, en donde se encuentra correlacionada con el término de perturbación , ésta puede regresarse en función de variables exógenas y utilizando su valor predicho en la ecuación de interés. Suponiendo un modelo de la forma:

$$
Y_{1}=\alpha_{0}+\lambda_{1} Y_{2}+\lambda_{1} Z_{2}+\varepsilon
$$

en la cual claramente y son endógenas y es una variable exógena. La solución al sesgo del parámetro de interés radica en estimar en función de las variables (que no están correlacionadas con el error, en este caso con el hecho de habitar en el mismo hogar) para obtener una variable predicha que se pueda utilizar para la estimación de una segunda etapa.

Así mismo se busca ampliar la muestra y obtener estadísticos más precisos utilizando la técnica de combinación de corte transversal, esto con EMNV 2005.

\subsubsection{Ecuación de Mincer y eliminación de 'debilidad de ubicación’}

Inicialmente se estima una ecuación de Mincer (Heckman, Lochner \& Todd, 2005) para padres e hijos de cara a superar la problemática de 'debilidad de ubicación':

$$
W=f\left(\text { tiempo, mujer }, e d u, e d u^{2}, \text { rural, }\right)
$$

Donde W es logaritmo natural del salario anual, tiempo equivale a una medida de experiencia en el trabajo reportado medida en años, mujer es una variable dicótoma con valor igual a uno si la observación es de sexo femenino y cero si es masculino, $e d u, e d u 2$ es la educación medida en años y educación al cuadrado respectivamente, rural es una variable dicótoma que hace referencia a uno si la observación reside en área rural y cero si habita en área urbana.

Utilizando los valores predichos de la ecuación de Mincer y controlando para la edad del hijo (Núñez \& Miranda, 2007), se procede a la estimación de la elasticidad intergeneracional para los hombres mayores de 17 años con más de cinco meses de experiencia en su trabajo reportado, resultando ésta de 0.4445 .

\begin{tabular}{|c|c|c|}
\hline \multicolumn{3}{|c|}{ Estimación de primer modelo de MEI } \\
\hline Variable & Coeficiente & P valor \\
\hline lnw_hijo (salario del hijo) & Variable dependiente & \\
\hline $\begin{array}{l}\text { lnw_padre (salario del padre) } \\
\text { Error estándar }\end{array}$ & $\begin{array}{l}0.4445^{* * *} \\
(0.06022) \\
\end{array}$ & 0.000 \\
\hline $\begin{array}{l}\text { Edad } \\
\text { Error estándar }\end{array}$ & $\begin{array}{l}0.01579 * * * \\
(0.00435)\end{array}$ & 0.000 \\
\hline
\end{tabular}

Cuadro 2. Estimación de primer modelo de movilidad económica intergeneracional 


\begin{tabular}{|l|l|l|}
\hline $\begin{array}{l}\text { Constante } \\
\text { Error estándar }\end{array}$ & $\begin{array}{l}5.3947^{* * *} \\
(0.63347)\end{array}$ & \\
\hline R cuadrado & 0.0816 & \\
\hline Prob>F & 0.000 & \\
\hline Observaciones & 725 & \\
\hline $\begin{array}{l}\text { *Significativo al 90\%, }{ }^{* *} \text { Significativo al 95\%, }{ }^{* * *} \text { Significativo al 99\%. } \\
\text { Errores estándar en paréntesis }\end{array}$ & \\
\hline
\end{tabular}

Se puede ver que, una vez que se obvia el hecho de que el hijo y el padre convivan en el mismo hogar, el valor de la elasticidad intergeneracional es de 0.44. Sin embargo se tiene una debilidad con la muestra, ya que existen muy pocas observaciones y existe sesgo del parámetro estimado.

Con el fin de aumentar la muestra, obtener estimadores más precisos y pruebas estadísticas con mayor poder (Wooldridge, 1999), se realiza una estimación en combinación de corte transversal (Pooling data de EMNV 2005 y EMNV 2009), utilizando los valores predichos de la ecuación de Mincer y con los hombres mayores de 17 años con más de cinco meses de laborar en su empleo reportado.

Se utiliza una variable dicótoma que hace referencia a 1 si el 'grupo' de datos es del año 2009 y cero de lo contrario. La razón de ser para la utilización de una variable dicótoma viene dada por la utilización de los salarios en logaritmos; como es de esperarse que los salarios aumenten con el tiempo, al aplicar logaritmo la inflación queda capturada en el intercepto para el año 2009 (Wooldridge, 1999).

Cuadro 3. Estimación de segundo modelo de movilidad económica intergeneracional

\begin{tabular}{|l|l|l|}
\hline Estimación de segundo modelo de MEI & Coeficiente & P valor \\
\hline Variable & Variable dependiente & \\
\hline lnw_hijo (salario del hijo) & $\begin{array}{l}0.49700^{* * *} \\
(0.04400)\end{array}$ & 0.000 \\
\hline $\begin{array}{l}\text { lnw_padre (salario del padre) } \\
\text { Error estándar }\end{array}$ & $\begin{array}{l}0.43781^{* * *} \\
(0.04554)\end{array}$ & 0.000 \\
\hline $\begin{array}{l}\text { Dicótoma } \\
\text { Error estándar }\end{array}$ & $\begin{array}{l}0.01806^{* * *} \\
(0.00294)\end{array}$ & 0.000 \\
\hline $\begin{array}{l}\text { Edad } \\
\text { Error estándar }\end{array}$ & $\begin{array}{l}4.3608^{* * *} \\
(0.43452)\end{array}$ & \\
\hline $\begin{array}{l}\text { Constante } \\
\text { Error estándar }\end{array}$ & 0.2630 & \\
\hline R cuadrado & 0.000 & \\
\hline Prob>F & 1,568 & \\
\hline $\begin{array}{l}\text { Observaciones } \\
\text { *Significativo al 90\%, }{ }^{* *} \text { Significativo al } 95 \%,{ }^{* * *} \text { Significativo al } \\
99 \% \text {. Errores estándar en paréntesis }\end{array}$ & \\
\hline
\end{tabular}


En la estimación se puede apreciar una elasticidad intergeneracional de 0.49. Esto se interpretaría como la situación en que padres con ingresos un $10 \%$ por debajo de la media de ingresos tienen hijos que se encuentran $4.9 \%$ por debajo de la media. Esto se traduce en persistencia en los ingresos, por lo que si un padre es pobre es de esperarse que el hijo también lo sea. En otras palabras, el $49 \%$ de las diferencias en los ingresos de los padres se transmiten a los hijos, evidenciándose persistencia intergeneracional y poca movilidad entre las generaciones.

Pero la estimación aún presenta el parámetro de elasticidad intergeneracional sesgado, dado que el modelo en teoría presenta autocorrelación y tiene a la variable dependiente como variable independiente y rezagada.

\subsubsection{Estimación por variable instrumental}

La estimación a través del uso de variables instrumentales resulta útil al existir correlación entre una variable explicativa (independiente) y el término de error o perturbación. En la segunda estimación existe autocorrelación y la variable dependiente es incluida con un rezago en las variables explicativas.

$$
\begin{aligned}
\text { En la ecuación a estimarse: } & \log y_{t}=\alpha_{0}+\delta_{1} \log y_{t-1}+\varepsilon_{T} \\
y_{t-1} \text { Contiene a }: \varepsilon_{t-1}: & y_{t-1}=\alpha_{0}+\delta_{1} y_{t-2}+\varepsilon_{t-1}
\end{aligned}
$$

O sea que el ingreso del padre se encuentra correlacionado con su propia dotación de capital humano, y el modelo teórico permite la existencia de autocorrelación porque la dotación de capital humano del padre puede transmitirse a la del hijo o, dicho de otra manera, el capital humano del hijo se encuentra correlacionado con el capital humano del padre, llevando esto a que el estimador referente a la elasticidad intergeneracional se encuentre sesgado.

La solución a la problemática del sesgo se puede resolver utilizando una Variable Instrumental (IV), método de estimación que reconoce la correlación entre las variables independientes y el término de error. La lógica detrás de la estimación a través de IV radica en obtener una variable que no se encuentre correlacionada con el término de error y que esté correlacionada con la variable independiente que se encuentra correlacionada con la perturbación.

En una muestra de la población (EMNV 2009) se puede probar si una variable es útil o no para ser una IV mediante dos maneras complementarias. Primero, la variable en cuestión no debe estar correlacionada con el error, es decir la dotación de capital humano. En este sentido se debe confiar en que no existe correlación debido a que es imposible una comprobación por no conocer la población. La covarianza entre y nunca puede ser probada, se debe mantener este supuesto en base al comportamiento económico o el sentido común (Wooldridge, 1999).

La correlación entre la variable independiente y la variable instrumental debe ser comprobada estimando una regresión entre la variable regresora que se busca remplazar, y la variable instrumental. El parámetro que precede a la potencial variable instrumental debe ser estadísticamente diferente de cero en una estimación 
de la forma:

$$
y_{t-1}=\alpha_{0}+\delta_{1} Z+u
$$

La variable instrumental que se utilizó es el valor del pago por alquiler efectivo e hipotético que realiza (o realizaría) el jefe de hogar por la vivienda en donde habita su hogar. ${ }^{7}$ La variable escogida no se encuentra correlacionada con la dotación de capital humano del padre o del hijo, es independiente de ésta. Así mismo la IV resulta ser muy completa pues se asume que el jefe de hogar realiza (o realizaría) el pago del alquiler en función de su ingreso de largo plazo. Si el ingreso de largo plazo fuera superior, entonces el jefe de hogar estaría dispuesto a pagar más por el alquiler de la vivienda y por una vivienda más favorable.

De la misma manera que en el modelo estimado previamente se utiliza la técnica de combinación de corte transversal con el fin de aumentar la muestra y obtener estadísticos más poderosos. La estimación en combinación de corte transversal se complementa con la inclusión de la variable instrumental.

Utilizando el salario del hijo y la variable instrumental, aislando el problema de 'ubicación', y combinando las bases de datos de corte transversal 2005 y 2009, se obtiene el siguiente resultado para trabajadores mayores de 17 años, de sexo masculino y con más de cinco meses laborando en su trabajo primario reportado:

Cuadro 4. Estimación de tercer modelo de movilidad económica intergeneracional

\begin{tabular}{|l|l|l|}
\hline Estimación de tercer modelo de MEI & Coeficiente & P valor \\
\hline Variable & Variable dependiente & - \\
\hline lnw_hijo (salario del hijo) & $\begin{array}{l}0.29000^{* * *} \\
(0.019764)\end{array}$ & 0.000 \\
\hline $\begin{array}{l}\text { IV } \\
\text { Error estándar }\end{array}$ & $\begin{array}{l}0.425530^{* * *} \\
(0.0414)\end{array}$ & 0.000 \\
\hline $\begin{array}{l}\text { Dummy } \\
\text { Error estándar }\end{array}$ & $\begin{array}{l}6.8679^{* * *} \\
(0.17586)\end{array}$ & 0.000 \\
\hline $\begin{array}{l}\text { Constante } \\
\text { Error estándar }\end{array}$ & $\begin{array}{l}0.01263^{* * *} \\
(0.00289)\end{array}$ & 0.000 \\
\hline $\begin{array}{l}\text { Edad } \\
\text { Error estándar }\end{array}$ & 0.2957 & \\
\hline R cuadrado & 0.000 & \\
\hline Prob>F & 1,551 & \\
\hline Observaciones & \\
\hline $\begin{array}{l}* \text { Significativo al 90\%, } \\
\text { Errores estándar en paréntesis) }\end{array}$ & & \\
\hline
\end{tabular}

7 Si el jefe de hogar efectivamente alquila una vivienda para la convivencia de su familia se utiliza dicho gasto. La EMNV también permite cuantificar el valor que pagaría el jefe del hogar por la vivienda en donde se habita si el hogar no alquila y tiene su propio hogar. En este sentido el jefe de hogar reporta una capacidad de pago en base a sus ingresos. 
La última estimación presenta una elasticidad intergeneracional de 0.29 , lo que equivale a decir que padres con ingresos un $10 \%$ por debajo de la media de ingresos tienen hijos que se encuentran $2.9 \%$ por debajo de la media de ingresos una generación posterior. Esto se traduce en persistencia en los ingresos, por lo que un padre hereda inercia en la generación de ingresos hacia el hijo, y evidencia que en la economía nicaragüense aún queda una tarea por cumplir en términos de generación de oportunidades para que los individuos nacidos en pobreza logren escalar en el escalafón de ingresos.

Cabe resaltar que el cálculo de los intervalos de confianza para el parámetro de interés indica que la elasticidad intergeneracional puede encontrarse en el rango de 0.25 y 0.33 al $95 \%$ de significancia.

Al comparar la elasticidad intergeneracional con estimaciones de economías más desarrolladas, como Chile (elasticidad de 0.52 -0.57) y EE.UU (0.5 0.6), se establece que en Nicaragua existe mayor movilidad intergeneracional a pesar de la notable persistencia en la generación de ingresos.

Los resultados obtenidos se encuentran en coherencia con los resultados estimados por estudios del Banco Mundial (Paes de Barro et al. 2008), en donde se establece que Nicaragua es uno de los países latinoamericanos que presenta puntajes bajos en el Índice de Oportunidades Humanas debido a la baja y dispareja cobertura de servicios básicos tales como asistencia a la escuela, y acceso a agua y saneamiento.

\section{Conclusiones y recomendaciones}

Es posible apreciar la existencia de una economía altamente desigual en la distribución de los ingresos, lo que representa disparidades en la capacidad de acceder a recursos como educación, salud, vivienda y nutrición. Se ha demostrado que dicha desigualdad es persistente a través de una generación, dado que las estimaciones de la elasticidad económica intergeneracional indican la existencia de una persistencia en la capacidad de generación de ingresos.

Básicamente, un padre de familia que en el periodo $t-1$ se encuentre un $10 \%$ por debajo del promedio de los ingresos engendrará un hijo que en su edad adulta generará ingresos un $2.9 \%$ por debajo del promedio de ingresos.

La elasticidad intergeneracional, para poder ser analizada en su totalidad, debe ser una medida calculable recurrentemente. En este sentido, se apuntaría a analizar el comportamiento de la movilidad a lo largo del tiempo, dando así mayor capacidad de entendimiento sobre la temática. Se recomienda una homogeneización en la metodología de captura de las diferentes fuentes de variable ingresos y acceso a servicios básicos en las distintas encuestas de hogares por parte del INIDE con el objetivo de aumentar precisión en variables como ingreso, gasto, acceso a recursos básicos, etc.

De igual manera se recomienda un desplazamiento del debate preponderante sobre al reducción de la desigualdad de los ingresos (desigualdad de resultado) hacia un debate más equilibrado que signifique coherencia entre el abordaje de la desigualdad de oportunidades (mayor y mejor acceso a educación, acceso a salud, seguridad alimentaria) y la desigualdad en la distribución de los ingresos. Ambas 
implican mayor accionar por parte del Estado, sobre todo utilizando políticas fiscales a través de dos de sus funciones básicas: la redistribución y la asignación de recursos escasos.

\section{Referencias bibliográficas}

Angrist, J. \& Krueger, A. (1992). The Effect of Age at School Entry on Educational Attainment: An Application of Instrumental Variables with Moments from Two Samples. Journal of American Statistical Association, 87 (418), 328-36

Arellano, M. \& Meghir, C. (1992). Female Labour Supply and On the Job Search: An Empirical Model Estimated Using Complementary Data Set. Review of Economic Studies, 59(3), 537-59.

Ayala Espino, J. (2000). Instituciones y Economía: Una introducción al neoinstitucionalismo económico. México: Fondo de Cultura Económica.

Bastiaensen, J., Flores, S., Steel, G., Sosa, C., Martinez, S. \& Alemán, M. (2011). Territorio, actores y estrategias de desarrollo. Cuaderno de Investigación (40) Managua: NITLAPAN.

Becker, G. \& Tomes, N. (1979). An Equilibrium Theory of the Distribution of Income and Intergenerational Mobility. Journal of Political Economy, (87), 1153-1189.

Corak, M. (2006). Do Poor Children Become Poor Adults? Lessons from a Cross Country Comparison of Generational Earnings Mobility. Bonn: Institute for the Study of Labor (IZA).

Debraj, R. (1998). Economía del Desarrollo. Barcelona: Anthony Bosch.

DeParle, J. (2012, enero, 4). Harder for Americans to Rise from Lower Rungs. New York Times Recuperado el 02 de junio de 2013, de http://www. nytimes.com/2012/01/05/us/harder-for-americans-to-rise-from-lower-rungs. html?pagewanted=all\&_r=0

Gary, B. \& Nigel, T. (1979). An Equilibrium Theory of the Distribution of Income and Intergenerational Mobility. Journal of Political Economy, 87, 53-89.

González, J. I. (2011). Utilitarismo y medición de la pobreza. Revista de Economía Institucional, 13(25), 89-103.

Heckman, J. J., Lochner, L. J. \& Todd, P. E. (2005). Earnings function, rates of return, and treatment effects: The Mincer equation and beyond. Massachusetts: National Bureau of Economic Research.

Instituto Nacional de Información del Desarrollo (2011a). Encuesta de Hogares sobre Medición del Nivel de Vida 2009: Aspectos Metodológicos. Managua: Autor. Recuperado el 2 de junio de 2013, de: http://www.inide.gob.ni/Emnv09/ Aspectos_Metodologicos.pdf

Instituto Nacional de Información del Desarrollo (2011b). ). Encuesta de Hogares sobre Medición del Nivel de Vida 2009: Bases de Datos. Managua: Autor.

Mayer, S. E. \& Lopoo, L. M. (2004). What do Trends in the Intergenerational Economic Mobility of Sons and Daughters in the United States Mean?. In M. Corak (Ed.). Generational Income Mobility in North America and Europe. (pp. 90-121). Cambridge: Cambridge University Press. 
Mazumder, B. (2012, abril). Is intergenerational economic mobility lower now than in the past? Chicago Fed Letter (297). Recuperado el 3 de junio de 2013, de http://www.chicagofed.org/digital_assets/publications/chicago_fed_ letter/2012/cflapril2012_297.pdf

Núñez, J. \& Miranda, L. (2007). Recent findings on intergenerational income and educational mobility in Chile. Serie Documentos de Trabajo (244). Santiago: Departamento de Economía, Universidad de Chile.

Paes de Barro, R., Ferreira, F., Molina, J. \& Saavedra, J. (2008). Measuring Inequality of Opportunities in Latin America and the Caribbean. Washington, D.C.: Banco Mundial.

Sachs, J. (2012). The Lost Generations. Project Syndicate. recuperado el 04 de juniod de 2013, de http://www.project-syndicate.org/commentary/educationnutrition-and-health-care-are-the-best-investments-by-jeffrey-d-sachs.

Sen, A. K. (1999). Development as Freedom. Oxford: Oxford University Press.

Solon, G. (1992), Intergenerational Income Mobility in the United States. The American Economic Review, 82(3), 393-408

Solon, G. (2004). A model of intergenerational mobility variation over time and place. In M. Corak (Ed.). Generational Income Mobility in North America and Europe (pp. 38-48). Cambridge: Cambridge University Press.

Stiglitz. J. (2012). The price of inequality. Recuperado el 3 de junio de 2013, de www. youtube.com/watch? $=$ gKOJqnAET9A

Wooldridge, J. (1999). Introduction to Econometrics. Massachusetts: South-Western Pub. 


\section{Anexo de salida de modelo}

Estimación de modelo econométrico utilizando variable instrumental y combinación de corte transversal (para hombres mayores de 17 años y con más de cinco meses trabajando en su trabajo primario reportado).

\begin{tabular}{r|rrr} 
Source & SS & df & MS \\
\hline $\begin{array}{r}\text { Model } \\
\text { Residual }\end{array}$ & $\begin{array}{l}330.052752 \\
78.307602\end{array}$ & $\begin{array}{r}1547 \\
.508278993\end{array}$ \\
\hline Total & 1116.36035 & 1550 & .720232486
\end{tabular}

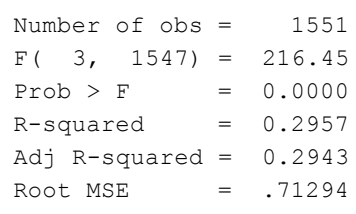

\begin{tabular}{r|rrrrrr}
\hline Inw_hijo & Coef. & Std. Err. & $t$ & P $>$ |t| & [95\% Conf. Interval] \\
\hline d & .4255303 & .0414076 & 10.28 & 0.000 & .3443092 & .5067513 \\
iv & .2900001 & .0197646 & 14.67 & 0.000 & .2512318 & .3287684 \\
edad & .0126334 & .0028917 & 4.37 & 0.000 & .0069613 & .0183055 \\
-cons & 6.867976 & .1758689 & 39.05 & 0.000 & 6.52301 & 7.212943 \\
\hline
\end{tabular}

Prueba de heterocedasticidad para estimación final. Se falla al rechazar la hipótesis nula sobre la existencia de homocedasticidad dado que la Prob $>$ chi2 $=0.1758$.

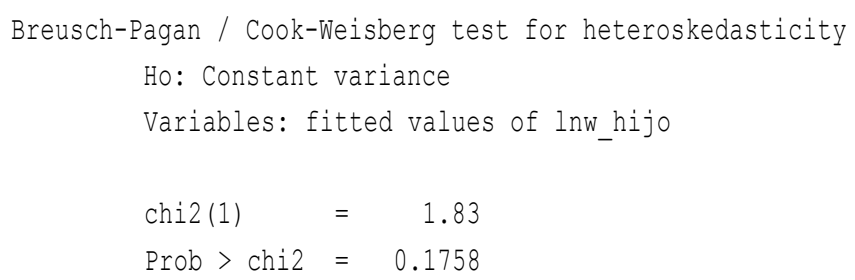

